\title{
Scientific Evidence for the Medical Use of Probiotics
}

\author{
Stephan C. Bischoffa ${ }^{\text {Martin Zeitz }}{ }^{\mathrm{b}}$ \\ ${ }^{a}$ Institute for Nutritional Medicine, University of Hohenheim, Stuttgart, and bepartment of Gastroenterology, \\ Infectious Diseases and Rheumatology, Charité - Campus Benjamin Franklin Berlin, Berlin, Germany
}

\section{Introduction}

Probiotics have been an issue of controversies as long as they exist. Discovered and firstly described by a few visionary scientists like Metchnikoff (France), Nissle (Germany) and Shirota (Japan) about a century ago, they were put on the market quite early on. However, for many years, probiotic usage was basically restricted to a few 'believers' and a kind of 'alternative physicians'. The reason for such restrictions was not just ignorance but also insufficient scientific evidence for this type of medicine. The protagonists were not always driven by scientific ambition but rather by practical aspects and personal experience of effectivity. The situation changed completely once evidence-based usage of probiotics was developed since end of the last century. A milestone work was that by Kruis et al., who published one of the first clinical trials on probiotics in Germany in 1997, the topic being the maintenance of remission in ulcerative colitis. This trial was definitively conducted according to all modern rules on how to perform a clinical study in a scientifically correct manner. Therefore, also the critics had to acknowledge such data. This opened a new level of discussion on probiotics and motivated many others to perform this kind of study. In the meantime, the body of probiotic literature has grown year by year. More than 1,000 clinical trials in man have been published on probiotics (source: PubMed, search terms: 'probiotics AND trial AND human', date: June 16th, 2010 , result: 1,180 hits), and only in $2009,1,713$ publications on probiotics can be found (search term: 'probiotics AND 2009 [year]'). Therefore, it is time to sum up and review the current evidence we have for the medical use of probiotics.

Danone Germany has taken up this idea and organized a scientific symposium entitled 'Probiotics in Health and Disease - What Is the Scientific Evidence?' held in Frankfurt a.M./Germany on December 11th, 2009. The symposium consisted of 4 keynote presentations, 5 workshops and a roundtable discussion, all performed by independent and highly respected scientists from Europe and the USA. The topics of the 4 presentations and the 5 workshops are presented in this special issue of Annals of Nutrition \& Metabolism. The presentations cover most of the important current issues on applied research in probiotics and include pros and cons of meta-analyses on probiotics (H. Szajewska), use of probiotics as treatment option in diarrhea (L.V. McFarland),

\section{KARGER}

Fax +4161306 1234

E-Mail karger@karger.ch

www.karger.com
(C) 2010 S. Karger AG, Basel

0250-6807/10/0575-0001\$26.00/0

Accessible online at:

www.karger.com/anm
Stephan C. Bischoff, MD

Institute for Nutritional Medicine, University of Hohenheim

DE-70593 Stuttgart (Germany)

Tel. + 4971145924 101, Fax + 4971145924343

E-Mail bischoff.stephan@uni-hohenheim.de 
Fig. 1. Participants of the roundtable discussion. From left to right: Martin Zeitz, Regina Fölster-Holst, Philip Rosenstiel, Nada Rayes, Stefan Meuer, Jürgen Schrezenmeir, Michael Blaut, Stephan C. Bischoff.

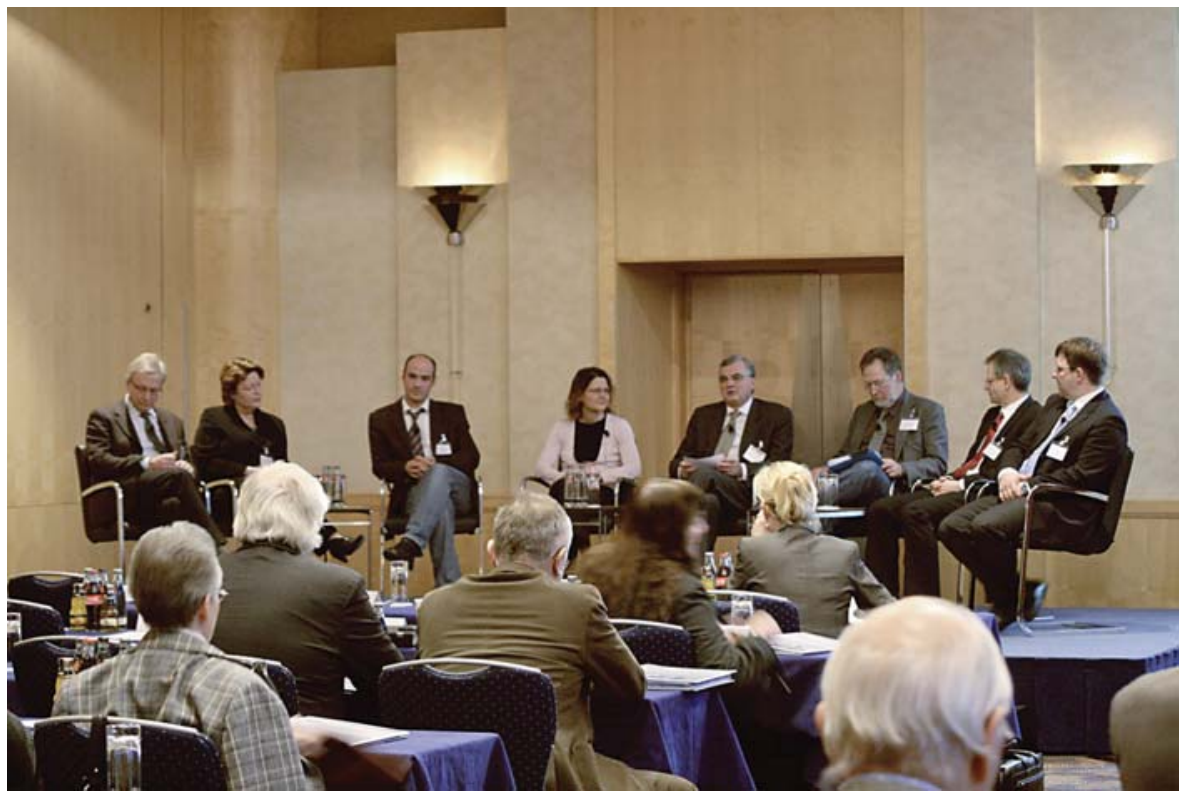

Table 1. Major clinical indications for the use of probiotics

Systematic reviews ( $>1,000$ patients) of randomized controlled trials ${ }^{1}$

$\square$ Acute gastroenteritis

$\square$ Clostridium-difficile-associated diarrhea

$\square$ Irritable bowel syndrome

$\square$ Necrotizing enterocolitis

Single randomized controlled trials

$\square$ Ulcerative colitis, remission phase

$\square$ Pouchitis

$\square$ Respiratory infections

$\square$ Bacterial infection in the perioperative phase of abdominal surgery

Single randomized controlled trials with controversial results

$\square$ Allergy

$\square$ Atopic dermatitis

${ }^{1}$ Adapted from Preidis and Versalovic [1].

irritable bowel syndrome (R.F. Meier) and prevention of necrosing enterocolitis (C.P. Braegger).

Major topics of current research in probiotics were discussed in the roundtable (moderated by S. Meuer) at the end of the meeting (fig. 1). In particular, the moderators of the 5 workshops presented the essentials of the discussion. It became clear that there is a gap between our clinical knowledge and our understanding of the mechanisms of action of probiotics. Despite this challenge, the present workshops focused mostly on the clinical issues, effectiveness of particular probiotics strains to prevent or treat particular diseases, and consequences regarding usage and recommendations. The overall positive message was that some areas for which probiotics usage is justified by criteria of evidence-based medicine can now be indicated (table 1, fig. 2). A couple of years before, most of us could only smile at such a statement. On the other hand, we are faced with many open questions, not only on mechanisms, but also on practical issues such as dosages, start and termination of therapy with probiotics, and, of course, on clear indications and definitions of population groups that benefit from it. Of special relevance is also the type of the probiotic organism, as all effects are strain specific. However, it is an advantage that new fields have been opened, e.g. the role of the gut microbiota for the development of obesity and the potential of probiotics to interfer which such mechanisms as positive modulators.

Research in the probiotic field is often complicated by the fact that probiotics are handled differently with regard to their legal status. Sometimes, probiotics are medical drugs, sometimes they are food supplements, sometimes both. The usage of a medical drug is restricted to prevention or therapy of disease and undergoes particular legal restrictions. A food supplement, however, is legally regulated in Europe by the 'health claim' 
regulations. Two possibilities can be envisioned, either 'body function claims' such as 'strengthens natural defense' or 'risk reduction claims', which means reduction of a risk factor, not of a disease. For example, LDL cholesterol could be a target for a risk reduction claim because this parameter is accepted as a relevant risk factor for arteriosclerosis. This legal discrimination of otherwise similar or even identical products affects research studies, accreditation trials, funding of trials and reimbursement issues. In particular, legal issues, and not only scientific issues, determine to a large extent the kind of study being performed. The consequence is that particular areas remain unclear, unsolved and unsatisfactory. Therefore, the aim of this symposium was also to identify gaps, which should be addressed in future investigator-initiated trials funded by governmental or commercial sources.

In workshop 1 'Probiotics and allergic diseases' (R. Fölster-Holst and E. Isolauri), a lot of conflicting data had to be discussed. On the one hand, there is perhaps no area for which so many promising findings on beneficial effects of probiotics exist. On the other hand, the data are so controversial that no general evidence-based recommendation can be made at present. Obviously, we must learn much more about the selection of relevant patient subgroups, of probiotic bacteria properties, of correct time windows in which studies are performed, etc. before we can generate more, and in particular more conclusive, studies in this field.

This reservation about recommendations of probiotics for the prevention of allergy and atopic dermatitis is surprising since even meta-analyses drawing positive conclusions exist. A possible reason for this could be that the inclusion criteria for such meta-analyses remained unclear and relevant trials with negative results have not been included. On the other hand, there are data sets showing amazingly positive results. Thus, there is still a clear rationale for the use of probiotics in the prevention of atopic disease although we cannot draw final conclusions at present. The practical consequences are easily confusing. On the one hand, general recommendations cannot be made, on the other no individual needs to be disadvised to take probiotics. If, for example, a pregnant mother of a family at risk of atopy asks if she can take probiotics to protect her baby against atopy, the answer can be yes, because there is a chance (not a certitude) that it helps and no relevant adverse effects have to be considered. In countries other than Germany, e.g. in Finland, this situation was already sufficient to phrase a recommendation for using probiotics

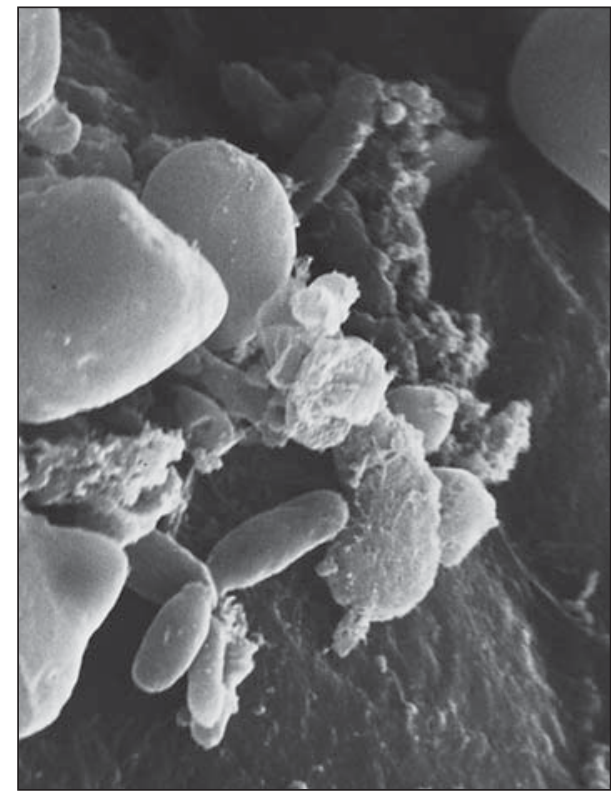

Fig. 2. Bacteria, starch granules and mucus remains on the surface of rat small intestinal epithelium (scanning electron micrograph; magnification: $\times 10,000)$. Source: Gastrointestinal Microbiology, DifE (German Institute of Human Nutrition).

for the prevention of atopic dermatitis in infants by treating the mothers of high-risk families, or young infants with early-life sensitization and multiple food allergies in the Finnish evidence-based medicine guideline.

The question arises as to why we have here the particular situation that the existing studies do not lack significance or power but yield diametrical results. This sounds like systematic errors or at least systematic differences that must be identified. Once identified, the conflicts might be solved quickly. Thus, the topic of probiotics and allergy remains exciting and new important findings are anticipated.

Workshop 2 'Probiotics and obesity' (M. Blaut and S.C. Bischoff) also addressed an interesting but more speculative and young research area, which at present is still far away from presenting definitive clinical recommendations. It has been convincingly reported, according to animal and human data, that obesity is associated with an altered gut microbiota and that the altered microbiota could be part of the etiology of weight gain, since it might change the digestion process towards enhanced energy extraction from food.

As usual in case of new research areas, we are confronted with many open questions. For example, the 
available studies differ with respect to methods, and the therapeutic consequences of the present findings are still unclear. Up to date, no probiotic trial in humans that shows a positive effect of probiotics in obese individuals on body weight or metabolic parameters is available. However, the rationale to do such kinds of research is rapidly increasing. Therefore, it is a matter of time until we can expect such trials.

Possibly, the anticipated beneficial effect of probiotics in the obese is not or not only based on modulation of food energy extraction and weight loss but also control of subclinical inflammation thought to trigger obesity-associated diseases like fatty liver disease or diabetes. These 2 anticipated effects could involve different probiotic strains. Clearly, more research is needed to clarify all these exciting questions.

Workshop 3 'Probiotics and respiratory diseases' (S. Meuer and J. Schrezenmeir) addressed extraintestinal effects of probiotics. Interestingly, we have meta-analyses showing that particular probiotics can reduce the duration and frequency of respiratory symptoms in the course of respiratory infections. However, consensus is lacking about the bacterial strain for which such a recommendation can be made. In addition, data are available showing that probiotics enhance antibody titers induced by vaccination, another mechanism by which probiotics might be useful in respiratory diseases. Possibly, this mechanism also provides an explanation why particular probiotics reduce symptoms of respiratory infections. Antibody enhancement might be induced not only during vaccination but also during wild-type infection. Interestingly, it has been shown that probiotics enhance not only classical IgG titers, but also IgA titers, e.g. in the course of rotavirus infection in children, a finding that could also have an impact on the prevention of allergic diseases. In future, other fields might be worked up, such as otitis media induced by streptococcus infection or halitosis caused by an altered mouth microbiome, for which we have only preliminary data at present.

In workshop 4 'Probiotics and gastrointestinal (GI) diseases' (P. Rosenstiel and E.F. Stange), the current knowledge on beneficial effects of selected probiotics for relapse prevention in ulcerative colitis and in pouchitis was discussed. Despite the impressing clinical results, we still do not know very much about the underlying mechanisms. Apart from irritable bowel disease, other GI diseases could benefit from probiotic therapy, e.g. travel diarrhea or irritable bowel syndrome, although the data are not as clear-cut as for ulcerative colitis. Possibly, we would need a more individual approach, a kind of 'personalized medicine', to improve the results in the field of probiotics. However, data combining outcome with e.g. genetic or microbiological analyses are hardly available so far.

Another field that needs further evaluation are infectious GI diseases. There are data showing positive effects of particular probiotics in Helicobacter pylori infection or resistance development; however, they are not homogeneous. The same is true for antibiotics-associated diseases, although the amount of positive data is much larger than for H. pylori infection. The 'personalized medicine' should be addressed not only with regard to the recipient of probiotics, but also concerning the probiotic strain used for therapy. Therefore, we need a much more detailed characterization of probiotic strains to understand the effects that can be expected. In summary, GI diseases such as ulcerative colitis, infectious diarrhea and irritable bowel syndrome are still the classical indications of the use of probiotics supported by large intervention trials and meta-analyses.

Workshop 5 'Probiotics in perioperative and intensive care medicine' (N. Rayes and P.B. Soeters) discussed the rationale to use probiotics in the critically ill patient. This exciting and complex field has to be divided into 2 areas.

In perioperative surgical patients the data on beneficial effects of particular probiotics are very convincing. Fifteen trials are available with overwhelming positive effects regarding prevention of bacterial infections, although different probiotic strains were used in these studies.

In the critically ill patients the data are much less clear. Actually, only data on patients with acute pancreatitis exist. Here we have 2 positive studies from Hungary and 1 negative study from the Netherlands showing enhanced mortality in the verum group. The reasons for the tremendous differences in results are still unclear. Most likely, methodological variation accounted for the opposing results. More experimental studies are needed before new clinical trials should be planned. Possibly, these severely ill patients are not the appropriate patient group for a rather preventive therapy concept like probiotics acting by immune modulation anyway. Indeed studies on other immune modulators such as glutamine or arginine and omega-3-fatty acids also revealed that they have a definitive benefit in basically healthy perioperative patients but not in severely ill patients with sepsis. Possibly, the latter patient group is just not appropriate for treatment with probiotics. Instead, the potential of probiotics to prevent infectious complications and other symptoms in less ill patients should be focused on, since 
we already have a convincing database for their usage in this field.

Clearly, we would like to learn more about the mechanisms mediating the positive clinical effects of probiotics, the microbiological properties of particular probiotic strains, e.g. why they act in an immunostimulatory and an immunosuppressive manner, and the relevant subgroups of recipients. Our knowledge on probiotics is now fascinating enough to be motivated to further improve their potential to maintain health and to prevent disease.

\section{Disclosure Statement}

S.C.B. is member of the scientific advisory board for Danone and has received lecture and/or consultancy fees from Ardeypharm, Danone, Nestle Nutrition, and Yakult - all Germany.

M.Z. has received lecture and/or consultancy fees from Danone, Germany.

Reference

1 Preidis GA, Versalovic J: Targeting the human microbiome with antibiotics, probiotics, and prebiotics: gastroenterology enters the metagenomics era. Gastroenterology 2009;136:2015-2031. 